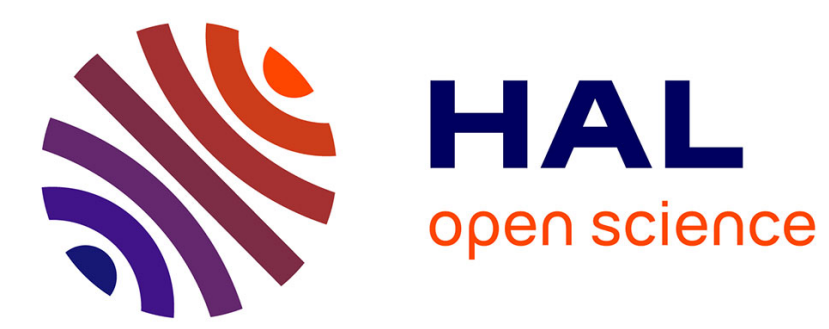

\title{
Aptitude des ondes de Rayleigh-Sezawa à caractériser l'adhérence
}

\author{
J. Pouliquen, B. Zouhri
}

\section{To cite this version:}

J. Pouliquen, B. Zouhri. Aptitude des ondes de Rayleigh-Sezawa à caractériser l'adhérence. Journal de Physique IV Proceedings, 1994, 04 (C5), pp.C5-753-C5-756. 10.1051/jp4:19945163 . jpa-00252843

\section{HAL Id: jpa-00252843 https://hal.science/jpa-00252843}

Submitted on 1 Jan 1994

HAL is a multi-disciplinary open access archive for the deposit and dissemination of scientific research documents, whether they are published or not. The documents may come from teaching and research institutions in France or abroad, or from public or private research centers.
L'archive ouverte pluridisciplinaire HAL, est destinée au dépôt et à la diffusion de documents scientifiques de niveau recherche, publiés ou non, émanant des établissements d'enseignement et de recherche français ou étrangers, des laboratoires publics ou privés. 


\section{Aptitude des ondes de Rayleigh-Sezawa à caractériser l'adhérence}

\section{J. POULIQUEN et B. ZOUHRI}

Laboratoire d'Acoustique-Ultrasons, Faculté Libre des Sciences, 13 rue de Toul, 59046 Lille cedex, France

The model studied includes three isotropic media : the cemented plate (plexiglass), the glue and the semi-infinite substrate (aluminium). We calculate the dispersion curve, the amplitudes and the energy repartition of the first three modes of Rayleigh-Sezawa waves (RSW). This study reveals the good sensitivity of RSW to characterize the sticking and the possibility to concentrate the energy in the glue.

\section{INTRODUCTION}

Les tentatives de caractériser l'adhérence par mesures acoustiques sont déjà nombreuses. Selon la nature du contact, glissant ou solide, ou dans le suivi de la prise d'une résine époxy, on détermine les variations, soit de la célérité et de l'atténuation soit du coefficient de réflexion des ondes utilisées [1 à 5].

Si on porte un intérêt particulier aux variations de célérité, les ondes de volume de cisaillement s'avèrent plus sensibles que les ondes de volume de compression; cependant, dans le meilleur des cas, ces variations n'excèdent guère $25 \%$ [5].

L'idée excellente de localiser l'énergie acoustique dans le film de colle par conversion d'ondes de Rayleigh en ondes de Lamb ou de Stoneley n'a donné que des variations de célérité de quelques pourcent [6].

L'emploi d'un autre type d'ondes, des ondes de Rayleigh-Sezawa, semble plus prometteur. Nous étudions un système à 3 milieux : lame collée, colle, substrat en fonction du coefficient de cisaillement de Lamé $\mu$ à différentes fréquences pour déterminer les zones de grandes sensibilités. Dans ces zones, le calcul des amplitudes et de l'énergie devrait montrer une "concentration acoustique" dans la colle [7].

\section{MODELE THEORIQUE}

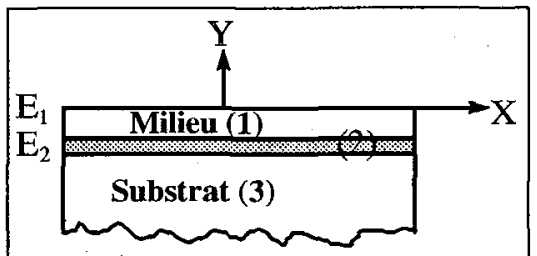

Fig. 1 Modèle théorique

Le système étudié comporte trois milieux supposés isotropes séparés par des plans paralleles: le milieu (1), la colle (2) et le substrat semi-infini (3) (Fig 1). Nous étudions une onde acoustique de surface qui s'y propage pour différentes conditions aux limites : conditions parfaites (continuité des contraintes et des déplacements) et conditions glissantes (continuité des contraintes et des déplacements normaux). Les contraintes à la surface libre restent toujours nulles.

Ces conditions appliquées au système donnent un système d'équations linéaires à seconds membres nuls; à chaque fréquence 
choisie l'annulation du déterminant foumit la courbe de dispersion des ondes étudiées. Comme pour les ondes de Lamb plusieurs modes existent à une même fréquence La connaissance de la célérité C permet le calcul des amplitudes et de l'énergie en chaque point ce qui donne la structure de l'onde.

La comparaison de la célérité $\mathrm{C}$ à celles des ondes de volume transversales $\mathrm{C}_{\mathrm{ti}}$ et longitudinales $\mathrm{C}_{\mathrm{li}}$ des milieux (i) permet de distinguer les types d'ondes obtenues : ondes Rayleigh si $\mathrm{C}<\mathrm{C}_{\mathrm{ti}}<\mathrm{C}_{\mathrm{li}}$, de RayleighSezawa si $\mathrm{C}_{\mathrm{ti}}<\mathrm{C}<\mathrm{C}_{\mathrm{li}}$ et de Sezawa si $\mathrm{C}_{\mathrm{ti}}<\mathrm{C}_{\mathrm{li}}<\mathrm{C}$.

Le modèle étudié est constitué d'une lame de plexiglas d'épaisseur $\mathrm{E} 1=100 \mu \mathrm{m}$ fixée par une lame de colle d'épaisseur $E_{2}=10 \mu \mathrm{m}$ au substrat d'aluminium. Le durcissement de la colle se faisant par polymérisation du monomère de plexiglas nous a amené à admettre que ce processus se réalisait à masse volumique $\rho$ et coefficient de Lamé $\lambda$ constants (d'autant que $\lambda$ agit peu sur la célérité), le second coefficient de Lamé $\mu$ croissant de 0 (liquide ou condition glissante) à $\mu_{m}$ (polymérisation achevée). La viscosité est négligée dans cette étude.

\section{RESULTATS}
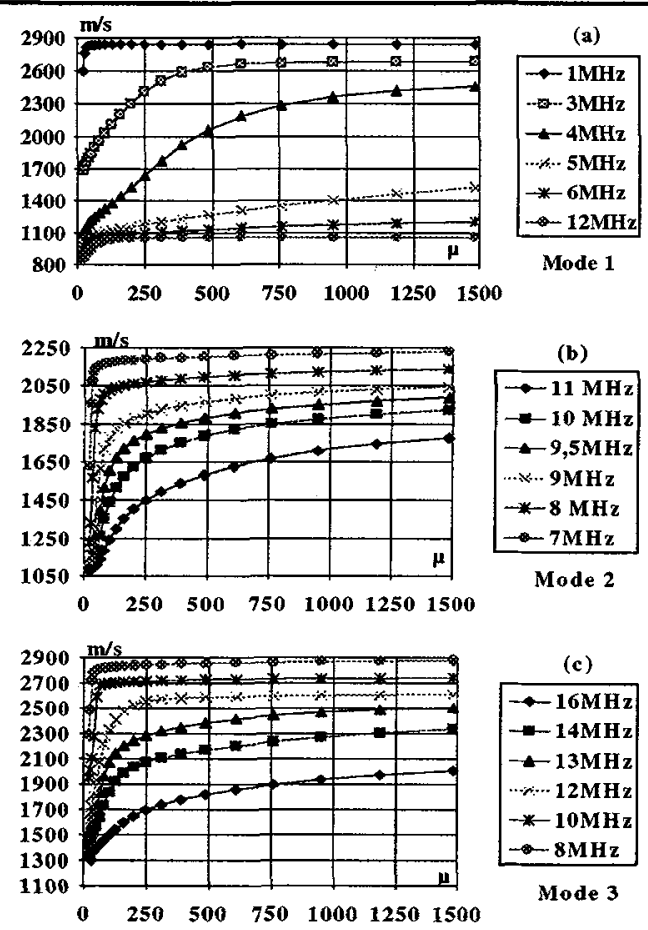

Fig. 2 Célérité en fonction de $\mu$ (MPa)

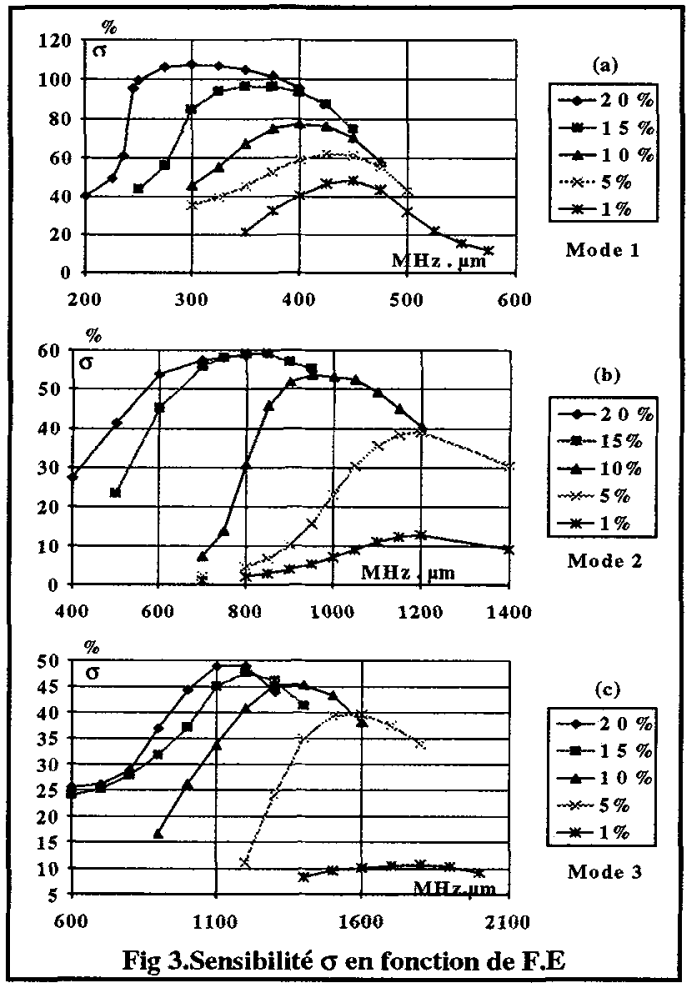

Les caractéristiques des matériaux utilisés sont, pour le socle d'aluminium : $\lambda=55200 \mathrm{MPa}$, $\mu=25900 \mathrm{MPa}, \rho=2700 \mathrm{~kg} / \mathrm{m}^{3}$ et pour le plexiglas : $\lambda=5447 \mathrm{MPa}, \mu=1483 \mathrm{MPa}, \rho=1180 \mathrm{~kg} / \mathrm{m}^{3}[5]$.

Les figures $2 \mathrm{a}, \mathrm{b} \& \mathrm{c}$ montrent les courbes de dispersion pour les trois premiers modes à plusieurs fréquences en fonction de $\mu$. Nous caractérisons l'aptitude à détecter l'adhérence par le coefficient de sensibilité : $\sigma=2\left(\mathrm{C}_{\max }-\mathrm{C}_{\min }\right) /\left(\mathrm{C}_{\max }+\mathrm{C}_{\min }\right)$ dont les variations avec $\mathrm{F} . \mathrm{E}_{1}$ \{fréquence $\mathrm{F}(\mathrm{MHz}) \mathrm{x}$ épaisseur du milieu (1) en $\mu \mathrm{m}$ \} sont données aux figures $3 \mathrm{a}, \mathrm{b} \& \mathrm{c}$ pour les épaisseurs relatives $\mathrm{E}_{2} / \mathrm{E}_{1}$ de colle mises en paramètres variant de 1 à $20 \%$ pour ces mêmes modes. La variation de $\sigma$ est d'autant plus grande que l'épaisseur de colle est importante et le premier mode s'avère le plus sensible.

Les amplitudes de vibrations dans les divers milieux sont calculees, aux fréquences où la sensibilite est maximale ou voisine du maximun, pour différentes valeurs de $\mu$ (Fig.4 \& 5). 
Les abscisses correspondent aux altitudes exprimées en longueur d'onde (E/A) et les ordonnées aux amplitudes longitudinales $\left(U / V_{1}\right)$ et transversales $\left(V / V_{1}\right)$ ramenées à l'amplitude transversale $V_{1}$ de la surface libre. En général, les amplitudes sont importantes dans la colle; il arrive même qu'elles soient beaucoup plus importantes que dans les plus proches régions des milieux (1) ou (3) (Fig 4c,d \& 5b, c). Ce fait invite à penser que nous nous trouvons dans une situation privilegiée pour détecter les défauts de collage. Pour ces mêmes valeurs de $\mu, \mathrm{F}$ et $\mathrm{E}_{2} / \mathrm{E}_{1}$, la répartition d'énergie est calculée (Fig 6 \& 7). Nous noterons une concentration d'énergie dans la colle ( milieu 2 ) (Fig 6c, d \& 7a, b).

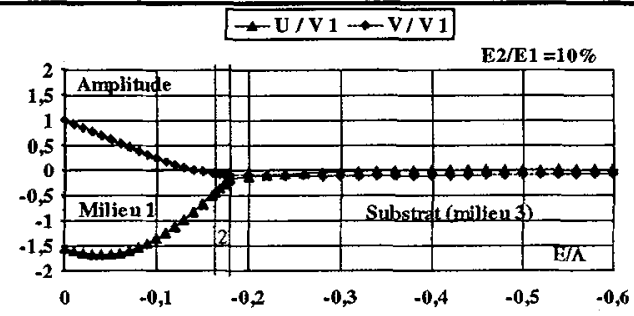

a) Mode $1(\mu=1483 \mathrm{MPa} F=4 \mathrm{MHz})$

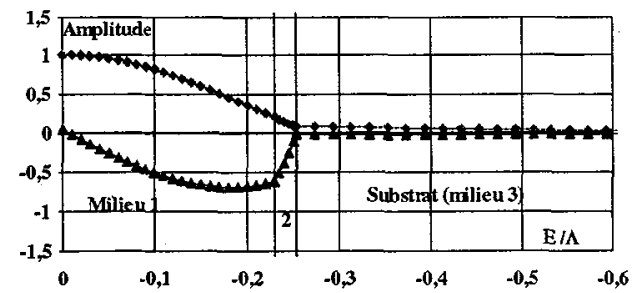

b) Aode $1(\mu=294 \mathrm{MPa} \mathrm{F}=4 \mathrm{MHz})$

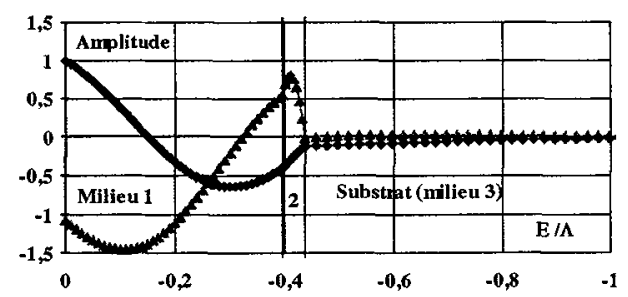

c) Mode $2(\mu=98.1 \mathrm{MPa} F=8 \mathrm{MHz})$

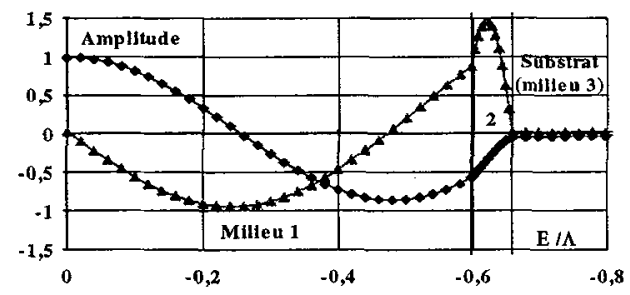

d)Mode $2(\mu=98.1: \mathrm{MPa} F=9.5 \mathrm{MHz})$

Fig.4 Ampl.long $\left(\mathrm{U} / \mathrm{V}_{1}\right) \&$ transv. $\left(\mathrm{V} / \mathrm{V}_{1}\right)$

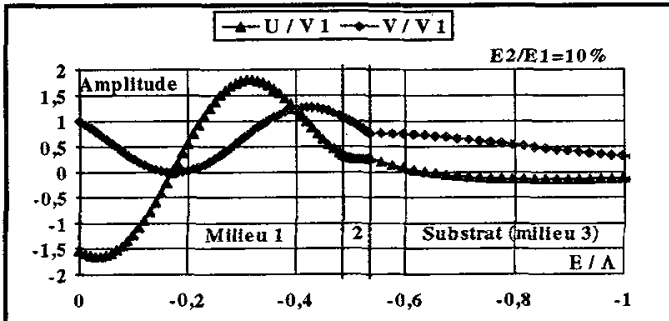

a) Mode 3 ( $\mu=1483 \mathrm{MPa} F=12.5 \mathrm{MHz}$ )

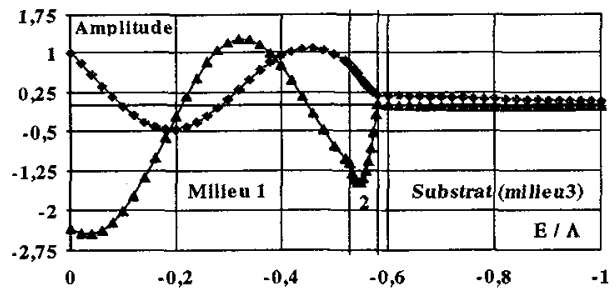

b) Mode 3 ( $\mu=196.2 \mathrm{MPa} F=12.5 \mathrm{MHz}$ )

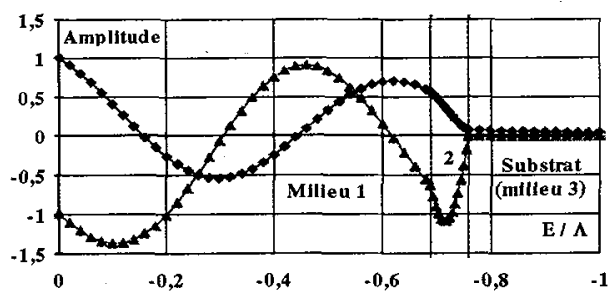

c) Mode 3 ( $\mu=196.2 \mathrm{MPa} F=14 \mathrm{MHz})$

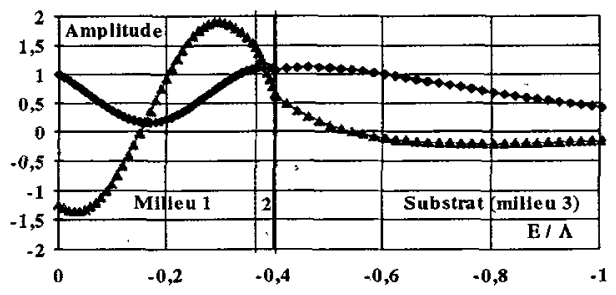

d) Mode 3 ( $\mu=490.5 \mathrm{MPa} F=14 \mathrm{MHz}$ )

Fig.5 Ampl.long $\left(U / V_{1}\right) \&$ transv. $\left(V / V_{1}\right)$

\section{CONCLUSION}

Cette étude révèle la bonne sensibilité des ondes de Rayleigh-Sezawa pour caractériser le collage et la possibilité de concentrer l'énergie acoustique dans la colle. Dans la limite de validité de ce simple modele, ce type d'onde semble attirant pour l'étude de l'adhérence. Cependant cet intêrêt reste lié à la possibilité d'exciter ces différents modes d'ondes. 


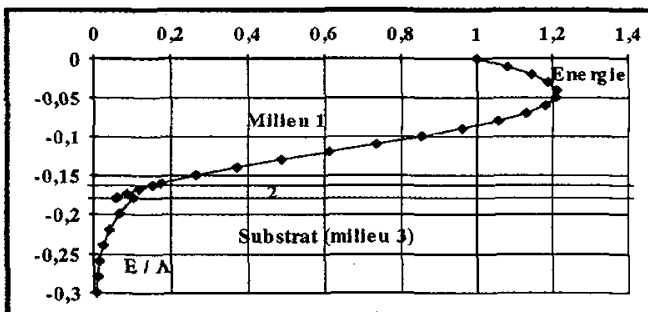

a) Mode $1(\mu=1483.3 \mathrm{MPa} F=4 \mathrm{MHz})$

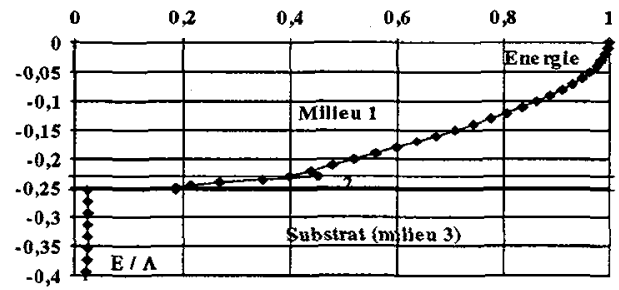

b) Mode 1 ( $\mu=294.3 M P a ~ F=4 M H z)$

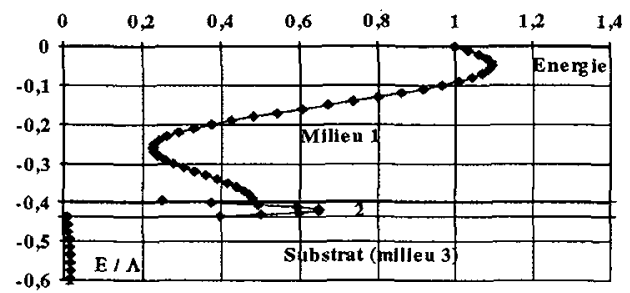

c) Mode $2(\mu=98.1 \mathrm{MPa} F=8 \mathrm{MHz})$

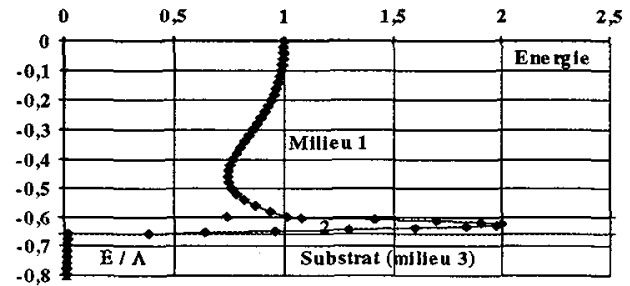

d) Mode 2 ( $\mu=98.1 \mathrm{MPa} F=9.5 \mathrm{MHz}$ )

Fig 6 Energie en fonction de $E / \Delta$

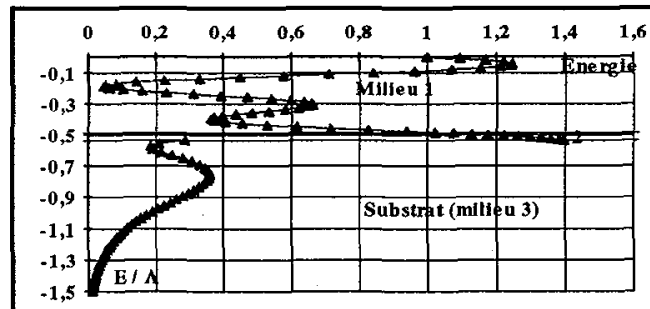

a) Mode 3 ( $\mu=1483.3 \mathrm{MPa} F=12.5 \mathrm{MHz}$ )

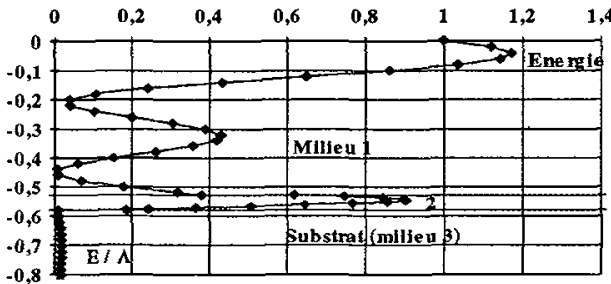

b) Mode 3 ( $\mu=196.2 \mathrm{MPa} F=12.5 \mathrm{MHz})$

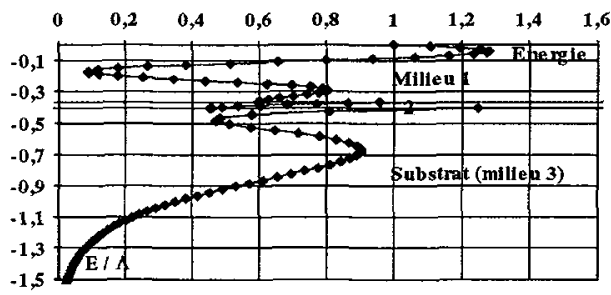

c) Mode $3(\mu=490.5 \mathrm{MPa} F=10 \mathrm{MHz})$

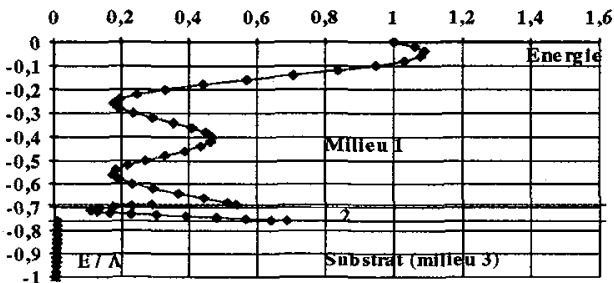

d) Mode 3 ( $\mu=196.2 \mathrm{MPa} F=14 \mathrm{MHz}$ )

Fig 7 Energie en fonction de $E / \Delta$

\section{Références}

[1] Pilarski A,Arch.of Acoust. 7 (1) (1982) 61-70

[2] Pilarski A and Rose J-L, J.Appl Phys.63 (2) (1988) 300-307

[3] Ouaftouh M. Thèse Universitê de Valenciennes (1990)

[4] Léomy F. Thèse Université de Paris (1990)

[5] Benel Moustafa Y. Thèse Université de Compiègne (1990)

[6] Rockhlin, Hefets M. and Rosen M., J.Appl. Phys.52(4) (1981) 2847-2851

[7] Moukala L-M. Thèse Université de Lille (1992) 